\title{
Prediction of Functional Outcome in Axonal Guillain-Barre Syndrome
}

\author{
Eun Jung Sung, MD, Dae Yul Kim, MD, Min Cheol Chang, MD, Eun Jae Ko, MD
}

Department of Rehabilitation Medicine, Asan Medical Center, University of Ulsan College of Medicine, Seoul, Korea

Objective To identify the factors that could predict the functional outcome in patients with the axonal type of Guillain-Barre syndrome (GBS).

Methods Two hundred and two GBS patients admitted to our university hospital between 2003 and 2014 were reviewed retrospectively. We defined a good outcome as being "able to walk independently at 1 month after onset" and a poor outcome as being "unable to walk independently at 1 month after onset". We evaluated the factors that differed between the good and poor outcome groups.

Results Twenty-four patients were classified into the acute motor axonal neuropathy type. There was a statistically significant difference between the good and poor outcome groups in terms of the GBS disability score at admission, and GBS disability score and Medical Research Council sum score at 1 month after admission. In an electrophysiologic analysis, the good outcome group showed greater amplitude of median, ulnar, deep peroneal, and posterior tibial nerve compound muscle action potentials (CMAP) and greater amplitude of median, ulnar, and superficial peroneal sensory nerve action potentials (SNAP) than the poor outcome group.

Conclusion A lower GBS disability score at admission, high amplitude of median, ulnar, deep peroneal, and posterior tibial CMAPs, and high amplitude of median, ulnar, and superficial peroneal SNAPs were associated with being able to walk at 1 month in patients with axonal GBS.

Keywords Guillain-Barre syndrome, Functional outcome, GBS disability score, MRC sum score, Acute motor axonal neuropathy

\section{INTRODUCTION}

Guillain-Barre syndrome (GBS) is a condition characterized by an acute or subacute onset of varying degrees of weakness in the limbs or cranial nerve-innervated muscles, associated with decreased or absent deep tendon reflexes, and a characteristic profile in the cerebrospinal fluid and electrophysiologic studies [1]. Advances in general care facilities and the availability of specific treatments have improved the outcome for patients with

Received April 29, 2015; Accepted October 12, 2015

Corresponding author: Dae Yul Kim

Department of Rehabilitation Medicine, Asan Medical Center, University of Ulsan College of Medicine, 88 Olympic-ro 43-gil, Songpa-gu, Seoul 05505, Korea. Tel: +82-2-3010-3800, Fax: +82-2-3010-6964, E-mail: dykimsmart@gmail.com

ORCID: Eun Jung Sung (http://orcid.org/0000-0002-3798-8978); Dae Yul Kim (http://orcid.org/0000-0003-1275-1736); Min Cheol Chang (http://orcid. org/0000-0002-7629-7213); Eun Jae Ko (http://orcid.org/0000-0001-7198-5407).

() This is an open-access article distributed under the terms of the Creative Commons Attribution Non-Commercial License (http://creativecommons.org/ licenses/by-nc/4.0) which permits unrestricted noncommercial use, distribution, and reproduction in any medium, provided the original work is properly cited. Copyright $\odot 2016$ by Korean Academy of Rehabilitation Medicine 
GBS. Nonetheless, approximately $20 \%$ of patients die from complications associated with GBS or remain severely disabled as a result of this condition [2,3].

The concept of GBS changed substantially in the 1990s because an axonal subtype of this disorder, acute motor axonal neuropathy (AMAN), was identified in northern China [4]. Consequently, GBS could be divided into two major subtypes, acute inflammatory demyelinating polyneuropathy (AIDP) and AMAN $[5,6]$. The pattern and speed of progression differ between AMAN and AIDP. Significantly shorter mean periods from onset to peak of illness are seen in AMAN [7]. Traditionally, AMAN has been thought to have a poorer clinical outcome $[8,9]$. However, some recent reports have shown that patients with AMAN can also achieve rapid recovery. In contrast to the relatively uniform speed of recovery in patients with AIDP, two different patterns of recovery are seen in patients with AMAN. Some recover within months; whereas, others have a slow and poor recovery [10-12]. Rapid recovery is caused by resolution of the conduction block, and poor recovery is associated with extensive axonal degeneration at the nerve roots [13].

Previous studies showed that preceding infection, age, rapid progression, disability at the nadir, disability at 2 weeks after entry, and electrophysiological characteristics are associated with long-term prognosis [3,14-19]. However, most of the studies assessing the prognostic factors in GBS were performed mainly in Europe and North America, where a diagnosis of AMAN is made in only $3 \%-17 \%$ of cases of GBS whereas the AIDP subtype accounts for $69 \%-90 \%$ of patients [20-22]. Notably, higher proportions of patients with GBS in East Asia and South America are classified into the axonal type [23,24]. This geographic difference in the proportion of the two disease subtypes suggests that prior studies that assessed the prognostic factors of GBS were based primarily on AIDP patients.

Unlike AIDP, in the AMAN subtype of GBS, autoimmune attack occurs at the nodes first and then extends to the paranodes [25]. The immunologic target of axonal and demyelinating GBS is different; hence, the prognostic factors may differ between these two types of GBS. However, virtually no research has been conducted to assess the factors associated with rapid recovery in patients with the axonal type of GBS. The aim of our current study was to identify the factors that can predict the functional outcomes for patients with axonal GBS.

\section{MATERIALS AND METHODS}

\section{Study design}

Two hundred and two patients admitted to our university hospital between January 2003 and December 2014 were retrospectively reviewed according to the following criteria: (1) above 18 years of age; (2) first occurrence of GBS; (3) fulfilled the National Institute of Neurological Disorders and Stroke diagnostic criteria for GBS [26]; (4) fulfilled the Van den Bergh criteria for the axonal type of GBS [27]; and (5) classified as having acute motor axonal neuropathy. Patients who were previously diagnosed with other peripheral neuropathies were excluded. We defined a poor outcome as the inability to walk independently at 1 month after admission (GBS disability score $\geq 3$ ) and a good outcome as being able to walk independently at 1 month after admission (GBS disability score $\leq 2$ ). These measures have been used as primary endpoints in many other previous studies on GBS [3,28-30].

Classification of axonal GBS according to the van den Bergh criteria

In the Van den Bergh criteria, the diagnosis of AIDP is supported by $>70 \%$ degree of slowing of motor conduction velocity below the lower limit of normal values, $>150 \%$ prolongation of motor distal latency above the upper limit of normal values, $>120 \%$ prolongation of the F latency above the upper limit of its normal value (and $>150 \%$ prolongation, if the distal negative peak compound muscle action potential [CMAP] amplitude was $<80 \%$ of the lower limit of normal values), or abnormal temporal dispersion (i.e., $>30 \%$ negative peak CMAP duration increase) in two or more nerves. For the axonal form of GBS, there should be no features of AIDP and distal CMAP should be $<80 \%$ of the lower limit of its normal value in two nerves.

\section{Data collection}

Data regarding patient age, gender, type of preceding infection, interval from infection to symptom onset, interval from symptom onset to intravenous gamma-globulin (IVIg) injection, cranial nerve dysfunction, sensory disturbance, cerebrospinal fluid protein, and respiratory disturbance requiring mechanical ventilation were re- 
viewed. In addition, Medical Research Council (MRC) sum scores and GBS disability scores at admission and 1 month after admission were documented. The MRC sum score is defined as the sum of MRC scores of six different muscles measured bilaterally, which results in a sum score ranging from 0 (quadriplegic) to 60 (normal) [31]. The GBS disability score is a functional scale for patients with GBS, ranging from 0 (normal) to 6 (death) [32]. Two points in the GBS disability score indicates an ability to walk more than $10 \mathrm{~m}$ without assistance but an inability to run. Electrophysiologic data involving the amplitude of CMAP; conduction velocity; distal motor latency; minimal F wave latency; conduction block of the median, ulnar, deep peroneal, and posterior tibial nerves; and the amplitude of sensory nerve action potential (SNAP) of the median, ulnar, superficial peroneal, and sural nerves was included. Abnormal nerve conduction study measures were defined as those with amplitudes lower than, and slowed nerve conduction velocity relative to the standard values of our electrodiagnostic laboratory. Motor conduction block was defined as $>30 \%$ negative peak amplitude reduction of proximal CMAP [27] and the value was recorded as the ratio of the proximal to the distal CMAP amplitude. When a patient had more than one follow-up electrodiagnostic study, the recordings that were considered the most informative were utilized for the final electrodiagnosis and evaluation.

\section{Statistical analysis}

Data were analyzed using the Statistical Package for Social Sciences software package (SPSS ver. 18.0; SPSS Inc.,

Table 1. Electrophysiological classification of GBS patients

\begin{tabular}{lc}
\hline & No. (\%) \\
\hline AIDP & $100(49.5)$ \\
\hline AMAN & $24(11.9)$ \\
\hline AMSAN & $2(1.0)$ \\
MFS & $31(15.3)$ \\
Normal & $11(5.4)$ \\
\hline Equivocal & $34(16.8)$ \\
\hline Total & $202(100)$ \\
\hline
\end{tabular}

GBS, Guillain-Barre syndrome; AIDP, acute inflammatory demyelinating polyneuropathy; AMAN, acute motor axonal neuropathy; AMSAN, acute motor sensory axonal neuropathy; MFS, Miller-Fisher syndrome.
Chicago, IL, USA). The Mann-Whitney U test and chisquare test were used to analyze demographic, clinical, and electrophysiologic differences in the good and poor outcome groups. Statistical significance was determined at p-values $<0.05$.

\section{RESULTS}

We enrolled 202 GBS patients between 2003 and 2014. Twenty-six patients (13\%) were categorized into the axonal type (Table 1). Twenty-four patients were classified into the AMAN type, and two patients were classified into the AMSAN (acute motor sensory axonal neuropathy) type of GBS. From the baseline characteristics of the motor axonal GBS patients, the mean age at onset was 52.7 years. Twenty-five percent of these patients required

Table 2. Demographics of the motor axonal GBS patients at admission $(\mathrm{n}=24)$

\begin{tabular}{lc}
\hline & Value \\
\hline Age (yr) & $52.7 \pm 14.3$ \\
Gender & \\
$\quad$ Male & $13(54.2)$ \\
\hline Female & $11(45.8)$ \\
\hline Preceding infection & \\
$\quad$ GI infection & $11(45.8)$ \\
$\quad$ URI & $5(20.8)$ \\
$\quad$ Nonspecific & $2(8.3)$ \\
$\quad$ None & $6(25.0)$ \\
\hline $\begin{array}{l}\text { Interval from infection } \\
\text { to symptom onset (day) }\end{array}$ & $11.3 \pm 6.5$ \\
\hline Interval from symptom & \\
to IVIg injection (day) & $8.9 \pm 8.7$ \\
Sensory symptom & $12(50.0)$ \\
\hline Mechanical ventilation & $6(25.0)$ \\
\hline Cranial nerve abnormalities & $15(62.5)$ \\
\hline CSF fluid protein (mg/dL) & $45.3 \pm 26.7$ \\
\hline GBSS at admission & $3.4 \pm 1.0$ \\
\hline MRC at admission & $39.4 \pm 19.6$ \\
\hline
\end{tabular}

Values are presented as mean \pm standard deviation or number (\%).

GBS, Guillain-Barre syndrome; GI, gastrointestinal; URI, upper respiratory infection; IVIg, intravenous gammaglobulin; CSF, cerebrospinal fluid; GBSS, Guillain-Barre syndrome disability score; MRC, Medical Research Council sum score. 
mechanical ventilation. The mean GBS disability score at admission was 3.4, and the mean MRC sum score at admission was 39.4 (Table 2). At one month after admission, six patients (25\%) were able to walk for $10 \mathrm{~m}$ without assistance. Ten patients (42\%) needed assistance to walk, two patients (8\%) were bedridden, and six patients (25\%) needed respiratory support. After dividing the patients by their functional outcomes at 1 month, we evaluated the factors that differed between the good and poor outcome groups (Table 3). Significant differences were found in terms of GBS disability score at admission ( $p<0.01$ ), GBS disability score at 1 month $(\mathrm{p}<0.01)$, and the MRC sum score at 1 month $(\mathrm{p}=0.03)$. However, mechanical ventila- tion did not show a significant difference between the two groups $(\mathrm{p}=0.10)$.

An electrophysiologic assessment was performed at a mean time period of 6 days after the admission date. In this analysis, the good outcome group showed a greater amplitude of median ( $p=0.02)$, ulnar $(\mathrm{p}=0.03)$, deep peroneal $(p=0.02)$, and posterior tibial nerve CMAPs $(p<0.01)$ compared with the poor outcome group. In the sensory nerve conduction study, the good outcome group showed a higher amplitude of median $(\mathrm{p}=0.02)$, ulnar $(p<0.01)$, superficial peroneal SNAPs $(p=0.01)$ than the poor outcome group (Table 4).

Table 3. Comparison of the clinical factors between groups stratified by patient functional outcomes at 1 month

\begin{tabular}{|c|c|c|c|}
\hline & $\begin{array}{c}1-\text { mo GBSS } \leq 2 \\
(n=6)\end{array}$ & $\begin{array}{c}\text { 1-mo GBSS }>2 \\
(n=18)\end{array}$ & p-value \\
\hline Age (yr) & $52.0 \pm 5.8$ & $52.9 \pm 16.4$ & 0.74 \\
\hline Gender & & & 0.24 \\
\hline Male & 2 & 11 & \\
\hline Female & 4 & 7 & \\
\hline Preceding infection & & & 0.22 \\
\hline GI infection & 2 & 9 & \\
\hline URI & 3 & 2 & \\
\hline Nonspecific & 0 & 2 & \\
\hline None & 1 & 5 & \\
\hline Interval from infection to symptom onset (day) & $12.8 \pm 6.5$ & $10.8 \pm 6.6$ & 0.40 \\
\hline Interval from symptom to IVIg injection (day) & $6.8 \pm 4.7$ & $9.6 \pm 9.6$ & 0.87 \\
\hline Sensory symptom (+:-) & & & 0.35 \\
\hline$(+)$ & 4 & 8 & \\
\hline$(-)$ & 2 & 10 & \\
\hline Mechanical ventilation (+:-) & & & 0.10 \\
\hline$(+)$ & 0 & 6 & \\
\hline$(-)$ & 6 & 12 & \\
\hline Cranial nerve abnormalities (+:-) & & & 0.81 \\
\hline$(+)$ & 4 & 11 & \\
\hline$(-)$ & 2 & 7 & \\
\hline CSF fluid protein (mg/dL) & $38.5 \pm 10.5$ & $48.1 \pm 30.9$ & 1.00 \\
\hline GBSS at admission & $2.5 \pm 0.5$ & $6.7 \pm 0.9$ & $<0.01^{*}$ \\
\hline MRC at admission & $52.7 \pm 5.9$ & $35.0 \pm 20.6$ & 0.06 \\
\hline GBSS at 1 month & $1.7 \pm 0.5$ & $3.8 \pm 0.9$ & $<0.01^{*}$ \\
\hline MRC at 1 month & $50.0 \pm 7.7$ & $33.7 \pm 16.1$ & $0.03^{*}$ \\
\hline
\end{tabular}

Values are presented as the mean \pm standard deviation or number.

GI, gastrointestinal; URI, upper respiratory infection; IVIg, intravenous gammaglobulin; CSF, cerebrospinal fluid; GBSS, Guillain-Barre syndrome disability score; MRC, Medical Research Council sum score. ${ }^{*} \mathrm{p}<0.05$. 
Table 4. Comparison of electrophysiologic values between groups stratified by patient functional outcomes at 1 month

\begin{tabular}{|c|c|c|c|}
\hline & $\begin{array}{c}1-m o \text { GBSS } \\
(n=6)\end{array}$ & $\begin{array}{c}1 \text {-mo GBSS >2 } \\
(n=18)\end{array}$ & p-value \\
\hline Median CMAP amplitude (mV) & $6.87 \pm 4.10$ & $3.08 \pm 3.76$ & $0.02 *$ \\
\hline Median CMAP latency (ms) & $4.53 \pm 0.98$ & $4.23 \pm 0.99$ & 0.51 \\
\hline Median CMAP conduction velocity (m/s) & $53.98 \pm 4.62$ & $54.84 \pm 5.44$ & 0.77 \\
\hline Median F wave latency (ms) & $27.15 \pm 1.89$ & $27.99 \pm 2.82$ & 0.84 \\
\hline Median nerve conduction block (proximal/distal) & $95.95 \pm 4.04$ & $85.48 \pm 25.03$ & 0.15 \\
\hline Ulnar CMAP amplitude (mV) & $6.07 \pm 4.01$ & $2.38 \pm 2.99$ & $0.03^{*}$ \\
\hline Ulnar CMAP latency (ms) & $3.32 \pm 0.56$ & $3.61 \pm 1.12$ & 0.84 \\
\hline Ulnar CMAP conduction velocity $(\mathrm{m} / \mathrm{s})$ & $55.78 \pm 4.16$ & $57.04 \pm 7.15$ & 0.71 \\
\hline Ulnar nerve F wave latency (ms) & $28.14 \pm 2.60$ & $27.86 \pm 2.24$ & 1.00 \\
\hline Ulnar nerve conduction block (proximal/distal) & $88.87 \pm 8.83$ & $93.26 \pm 6.90$ & 0.28 \\
\hline Deep peroneal CMAP amplitude (mV) & $4.03 \pm 3.41$ & $1.05 \pm 1.33$ & $0.02^{*}$ \\
\hline Deep peroneal CMAP latency (ms) & $4.47 \pm 0.52$ & $5.09 \pm 1.63$ & 0.34 \\
\hline Deep peroneal CMAP conduction velocity (m/s) & $46.68 \pm 4.07$ & $42.49 \pm 5.43$ & 0.12 \\
\hline Deep peroneal nerve F wave latency (ms) & $49.58 \pm 1.63$ & $49.62 \pm 4.77$ & 0.62 \\
\hline Deep peroneal nerve conduction block (proximal/distal) & $87.32 \pm 6.23$ & $82.96 \pm 19.77$ & 0.68 \\
\hline Posterior tibial CMAP amplitude (mV) & $15.35 \pm 10.30$ & $3.51 \pm 4.08$ & $<0.01^{*}$ \\
\hline Posterior tibial CMAP latency (ms) & $4.18 \pm 0.50$ & $4.57 \pm 0.84$ & 0.35 \\
\hline Posterior tibial CMAP conduction velocity (m/s) & $44.68 \pm 3.33$ & $43.59 \pm 2.95$ & 0.56 \\
\hline Posterior tibial nerve F wave latency (ms) & $50.80 \pm 5.38$ & $50.91 \pm 4.32$ & 0.85 \\
\hline Posterior tibial nerve conduction block (proximal/distal) & $71.43 \pm 11.52$ & $72.03 \pm 23.18$ & 0.53 \\
\hline Median SNAP amplitude $(\mu \mathrm{V})$ & $23.28 \pm 6.57$ & $13.49 \pm 11.54$ & $0.02^{*}$ \\
\hline Ulnar SNAP amplitude $(\mu \mathrm{V})$ & $23.09 \pm 13.40$ & $11.06 \pm 8.39$ & $<0.01^{*}$ \\
\hline Superficial peroneal SNAP amplitude $(\mu \mathrm{V})$ & $10.85 \pm 3.77$ & $5.66 \pm 7.10$ & $0.01^{*}$ \\
\hline Sural SNAP amplitude $(\mu \mathrm{V})$ & $17.84 \pm 7.40$ & $12.25 \pm 12.28$ & 0.06 \\
\hline
\end{tabular}

Values are presented as the mean \pm standard deviation.

GBSS, Guillain-Barre syndrome disability score; CMAP, compound muscle action potential; SNAP, sensory nerve action potential.

${ }^{*} \mathrm{p}<0.05$.

\section{DISCUSSION}

In the present study, $75 \%$ of the AMAN patients were unable to walk independently at 1 month after admission. This is a larger number than that in a previous study of all GBS patients, which reported that $55 \%$ of these cases could not walk independently at 4 weeks after inclusion [29]. In the present study, a poor outcome showed a strong association with the GBS disability score at admission; amplitude of the CMAP of the median, ulnar, deep peroneal, and posterior tibial nerves; and amplitude of the SNAP of the median, ulnar, and superficial peroneal nerves. This indicates that the disease severity at admission and the extent of axonal injury expressed as a low amplitude in an electrophysiologic test may be predictive of the prognosis at 1 month after admission.

There has been some debate as to whether preceding diarrhea is a predictor of poor outcome in GBS. This finding was reported in five studies $[3,15,17,18,29]$ but not in two other reports $[14,16]$. In the present study, the presence of diarrhea before the onset of weakness was not statistically different between the two groups. This finding does not correspond with the well-established concept that Campylobacter jejuni-induced diarrhea and the axonal type of GBS infection are closely associated with each other. Our result can be explained by the fact that not only C. jejuni, but also other non-Campylobacter bacteria or viral infections could represent a common 
etiological basis of infectious diarrhea and GBS development. Even if $C$. jejuni-induced diarrhea was a negative prognostic factor, we could not determine whether diarrhea was associated with $C$. jejuni because we did not have the stool evaluation results. We could not distinguish $C$. jejuni-induced diarrhea from other nonspecific diarrheas. Non-specific diarrhea did not show any correlation with a poor prognosis in our results.

In contrast to other studies that included other types of GBS patients, old age was not found to be a prognostic factor in the present study. Two previous studies have reported that younger people are more likely to acquire a $C$. jejuni infection and develop the axonal type of GBS. Also, there has been an epidemic of axonal GBS among children in northern China [4], and a previous study has reported that young men living in rural areas in Bangladesh are mainly affected by the AMAN type of GBS [33]. However, this result is not consistent with our present findings. In our current study series, the mean age of GBS onset was 52.7 years, which is higher than that reported in other studies of GBS. Even though our present investigation included only a small number of AMAN patients, the standard deviation of age for axonal GBS is smaller than that in the other study's data. Although the issue of whether younger or older patients are more susceptible to axonal GBS is not yet clear, we speculated that if a narrower range of age groups was susceptible to axonal GBS than to the entire spectrum of GBS, age might not be a negative prognostic factor.

Cornblath et al. [34] described distal CMAP amplitude as the single best predictor of prognosis through electrodiagnostic testing in 210 of 245 GBS patients. Miller et al. [35] stated that most powerful predictor of a poor outcome in 60 severe GBS patients was a reduced mean CMAP amplitude. In other studies, less than $20 \%$ or $10 \%$ of the lower limit of normal was found to be more consistent with a poor prognosis. This is concordant with our present study, which showed that the amplitude of median, ulnar, deep peroneal, and posterior tibial CMAPs and the amplitude of median, ulnar, and superficial peroneal SNAPs were higher in the good prognosis group. Besides, the mean amplitude of the CMAP in the poor prognosis group was more than $48 \%$ of the lower limit of normal, and the mean amplitude of the SNAP was more than $68 \%$ of the lower limit of normal, a far greater value than was expected based on previous studies. A meth- odological problem may have affected the result. In the present study, we used the Mann-Whitney U test to identify the prognostic factors. The Mann-Whitney test uses the mean values for evaluating statistical significance. In axonal GBS patients, the involved nerve is unique to each individual; the mean amplitude of each nerve can become nonspecific when the value for each of the involved nerves closely correlated with the GBS disability score at 1 month is averaged.

Our present study had several limitations. First, we included a small number patients from our university hospital only. Second, our electrophysiologic evaluation periods were heterogeneous among individuals and most of our GBS patients were assessed through only one or two electrophysiologic studies. Because there were an insufficient number of serial evaluations for electrophysiologic studies, the lack of distinction between reversible conduction failure in the axonal type of GBS and demyelinating conduction block might have led to the incorrect classification of AMAN as AIDP. In another aspect, there is a possibility that patients with the severe demyelinating type of GBS were considered to have the axonal type of GBS. Third, the primary outcome in our patient series was not measured at 1 month after symptom onset, but it was measured at 1 month after admission. Fourth, antiganglioside antibody evaluations and microbiological examination results were not available. Because our study was performed retrospectively, there were also some limitations in terms of data availability. Finally, even though there were several statistically significant factors established between the two study groups by the chi-square and Mann-Whitney U tests, we could not identify any statistically significant factor by logistic regression analysis that was predictive of a poor outcome at 1 month.

The present study is the first study that attempted to identify the prognostic factors among adult patients with motor axonal GBS. Patients with a higher GBS disability score at admission and a low amplitude in the electrophysiologic study had an inability to walk independently at 1 month after admission. However, old age and preceding diarrhea did not correlate with a poor outcome in patients with axonal GBS. Electrophysiological evaluations and initial disease severity are thought to be important predictive factors of early recovery in patients with axonal GBS. Our findings are limited by the relatively 
small number of patients analyzed, and we were unable to provide more informative results because our analysis was performed retrospectively. Further complementary prospective studies that involve a larger number of cases and evaluate long-term outcomes are warranted.

\section{CONFLICT OF INTEREST}

No potential conflict of interest relevant to this article was reported.

\section{REFERENCES}

1. Hughes RA, Cornblath DR. Guillain-Barre syndrome. Lancet 2005;366:1653-66.

2. van Doorn PA. Treatment of Guillain-Barre syndrome and CIDP. J Peripher Nerv Syst 2005;10:113-27.

3. van Koningsveld R, Steyerberg EW, Hughes RA, Swan AV, van Doorn PA, Jacobs BC. A clinical prognostic scoring system for Guillain-Barre syndrome. Lancet Neurol 2007;6:589-94.

4. McKhann GM, Cornblath DR, Griffin JW, Ho TW, Li $\mathrm{CY}$, Jiang Z, et al. Acute motor axonal neuropathy: a frequent cause of acute flaccid paralysis in China. Ann Neurol 1993;33:333-42.

5. Hafer-Macko CE, Sheikh KA, Li CY, Ho TW, Cornblath DR, McKhann GM, et al. Immune attack on the Schwann cell surface in acute inflammatory demyelinating polyneuropathy. Ann Neurol 1996;39:625-35.

6. Hafer-Macko C, Hsieh ST, Li CY, Ho TW, Sheikh K, Cornblath DR, et al. Acute motor axonal neuropathy: an antibody-mediated attack on axolemma. Ann Neurol 1996;40:635-44.

7. Hiraga A, Kuwabara S, Ogawara K, Misawa S, Kanesaka T, Koga M, et al. Patterns and serial changes in electrodiagnostic abnormalities of axonal GuillainBarre syndrome. Neurology 2005;64:856-60.

8. Feasby TE, Gilbert JJ, Brown WF, Bolton CF, Hahn AF, Koopman WF, et al. An acute axonal form of GuillainBarre polyneuropathy. Brain 1986;109(Pt 6):1115-26.

9. Wee AS, Abernathy SD. The sural sensory nerve is usually spared in Guillain-Barre syndrome. J Miss State Med Assoc 2003;44:251-5.

10. Hiraga A, Mori M, Ogawara K, Hattori T, Kuwabara S. Differences in patterns of progression in demyelinating and axonal Guillain-Barre syndromes. Neurology
2003;61:471-4.

11. Hiraga A, Mori M, Ogawara K, Kojima S, Kanesaka T, Misawa S, et al. Recovery patterns and long term prognosis for axonal Guillain-Barre syndrome. J Neurol Neurosurg Psychiatry 2005;76:719-22.

12. Tamura N, Kuwabara S, Misawa S, Kanai K, Nakata M, Sawai S, et al. Time course of axonal regeneration in acute motor axonal neuropathy. Muscle Nerve 2007; 35:793-5.

13. Kuwabara S, Asahina M, Koga M, Mori M, Yuki N, Hattori T. Two patterns of clinical recovery in GuillainBarre syndrome with IgG anti-GM1 antibody. Neurology 1998;51:1656-60.

14. McKhann GM, Griffin JW, Cornblath DR, Mellits ED, Fisher RS, Quaskey SA. Plasmapheresis and GuillainBarre syndrome: analysis of prognostic factors and the effect of plasmapheresis. Ann Neurol 1988;23:347-53.

15. Visser LH, Schmitz PI, Meulstee J, van Doorn PA, van der Meche FG. Prognostic factors of GuillainBarre syndrome after intravenous immunoglobulin or plasma exchange. Dutch Guillain-Barre Study Group. Neurology 1999;53:598-604.

16. Chio A, Cocito D, Leone M, Giordana MT, Mora G, Mutani R, et al. Guillain-Barre syndrome: a prospective, population-based incidence and outcome survey. Neurology 2003;60:1146-50.

17. Hadden RD, Karch H, Hartung HP, Zielasek J, Weissbrich B, Schubert J, et al. Preceding infections, immune factors, and outcome in Guillain-Barre syndrome. Neurology 2001;56:758-65.

18. The prognosis and main prognostic indicators of Guillain-Barre syndrome: a multicentre prospective study of 297 patients. The Italian Guillain-Barre Study Group. Brain 1996;119(Pt 6):2053-61.

19. A prospective study on the incidence and prognosis of Guillain-Barre syndrome in Emilia-Romagna region, Italy (1992-1993). Emilia-Romagna Study Group on Clinical and Epidemiological Problems in Neurology. Neurology 1997;48:214-21.

20. Jacobs BC, van Doorn PA, Schmitz PI, Tio-Gillen AP, Herbrink P, Visser LH, et al. Campylobacter jejuni infections and anti-GM1 antibodies in Guillain-Barre syndrome. Ann Neurol 1996;40:181-7.

21. Rees JH, Soudain SE, Gregson NA, Hughes RA. Campylobacter jejuni infection and Guillain-Barre syndrome. N Engl J Med 1995;333:1374-9. 
22. Kuwabara S, Yuki N, Koga M, Hattori T, Matsuura D, Miyake M, et al. IgG anti-GM1 antibody is associated with reversible conduction failure and axonal degeneration in Guillain-Barre syndrome. Ann Neurol 1998;44:202-8.

23. Ho TW, Mishu B, Li CY, Gao CY, Cornblath DR, Griffin JW, et al. Guillain-Barre syndrome in northern China. Relationship to Campylobacter jejuni infection and anti-glycolipid antibodies. Brain 1995;118(Pt 3):597605.

24. Ogawara K, Kuwabara S, Mori M, Hattori T, Koga M, Yuki N. Axonal Guillain-Barre syndrome: relation to anti-ganglioside antibodies and Campylobacter jejuni infection in Japan. Ann Neurol 2000;48:624-31.

25. Kuwabara S, Yuki N. Axonal Guillain-Barre syndrome: concepts and controversies. Lancet Neurol 2013;12: 1180-8.

26. Asbury AK, Cornblath DR. Assessment of current diagnostic criteria for Guillain-Barre syndrome. Ann Neurol 1990;27 Suppl:S21-4.

27. Van den Bergh PY, Pieret F. Electrodiagnostic criteria for acute and chronic inflammatory demyelinating polyradiculoneuropathy. Muscle Nerve 2004;29:56574.

28. van der Meche FG, Schmitz PI. A randomized trial comparing intravenous immune globulin and plasma exchange in Guillain-Barre syndrome. Dutch Guillain-
Barre Study Group. N Engl J Med 1992;326:1123-9.

29. Walgaard C, Lingsma HF, Ruts L, van Doorn PA, Steyerberg EW, Jacobs BC. Early recognition of poor prognosis in Guillain-Barre syndrome. Neurology 2011;76: 968-75.

30. Double-blind trial of intravenous methylprednisolone in Guillain-Barre syndrome. Guillain-Barre Syndrome Steroid Trial Group. Lancet 1993;341:586-90.

31. Kleyweg RP, van der Meche FG, Schmitz PI. Interobserver agreement in the assessment of muscle strength and functional abilities in Guillain-Barre syndrome. Muscle Nerve 1991;14:1103-9.

32. Hughes RA, Newsom-Davis JM, Perkin GD, Pierce JM. Controlled trial prednisolone in acute polyneuropathy. Lancet 1978;2:750-3.

33. Islam Z, Jacobs BC, van Belkum A, Mohammad QD, Islam MB, Herbrink $\mathrm{P}$, et al. Axonal variant of Guillain-Barre syndrome associated with Campylobacter infection in Bangladesh. Neurology 2010;74:581-7.

34. Cornblath DR, Mellits ED, Griffin JW, McKhann GM, Albers JW, Miller RG, et al. Motor conduction studies in Guillain-Barre syndrome: description and prognostic value. Ann Neurol 1988;23:354-9.

35. Miller RG, Peterson GW, Daube JR, Albers JW. Prognostic value of electrodiagnosis in Guillain-Barre syndrome. Muscle Nerve 1988;11:769-74. 